\title{
Multimodale bildgebende Diagnostik bei Fisteln des Sinus piriformis
}

Die kongenitale Fistel des Sinus pirifomis (FSP) ist eine Anomalie, die auf die Obliteration des dritten und vierten Branchialbogens (Hsin et al, J R Coll Surg 1998; $43: 125$ - 126) zurückzuführen ist. Die potenzielle Verbindung mit dem Pharynx ist Voraussetzung zur Entwicklung von Entzündungsprozessen des Halses, am häufigsten in Form einer eitrigen Thyreoiditis. Diese Erkrankung ist selten. Sie betrifft vor allem Kinder und Jugendliche und umfasst 0,1\% der chirurgischen Interventionen an der Schilddrüse.

Klinische Merkmale und bilddiagnostische Methoden zum Nachweis der FSP werden bei vier jungen Patienten vorgestellt. Die anatomischen Voraussetzungen zur Entstehung des Entzündungsprozesses werden erläutert.

\section{Fallberichte}

Zwei Patienten mit akuter rezidivierender Thyreoiditis und zwei Patienten mit Halsabszess wurden in unserer Klinik untersucht. In allen Fällen wurden eine Hypopharyngographie mit Bariumsulfat, eine kontrastverstärkte Computertomographie und eine Sonographie durchgeführt.

\section{Fall 1}

Ein 6-jähriger Junge, der im Rahmen einer akuten Entzündung der Schilddrüse mit Antibiotika behandelt worden war, stellte sich ein Jahr später mit einer akuten Thyreoiditis vor. Klinisch zeigten sich ein vergrößerter und schmerzhafter linker Schilddrüsenlappen ohne Rötung, deutliches Ödem im linken Halsbereich und Fieber. Die Laboruntersuchungen ergaben eine beschleunigte BSG $(60 \mathrm{~mm} /$ $\mathrm{h})$, Leukozytose und normale Werte für $\mathrm{TSH}, \mathrm{FT}_{4} \mathrm{FT}_{3}$. In der Ultraschalluntersuchung des Halses zeigten sich eine hyporeflexive, schlecht abgrenzbare Zone im linken Vorderlappen der Schilddrüse und ein unauffälliges benachbartes Parenchym (Abb.1). Nach Antibiotikabehandlung trat eine deutliche Befundbesserung ein. Eine Kontrastmitteluntersuchung mit Bariumsulfat zeigte in der a.p.-Projektion eine Kontrastmittelansammlung im linken Recessus piriformis.

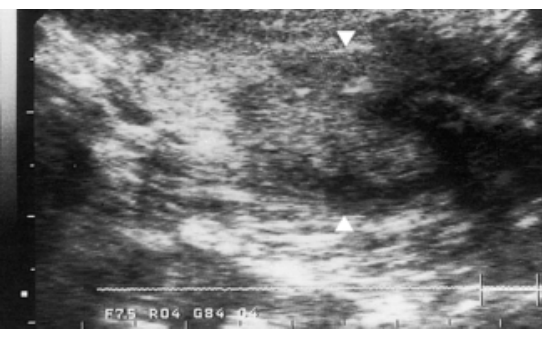

Abb. 1 Sonographie des Halses - hyporeflexive Zone li. Vorderlappen der Schilddrüse $(\triangleright 4)$.

Computertomographisch wurde eine hyperdense ca. $2 \mathrm{~mm}$ durchmessende Läsion mit hohen Dichtewerten (ca. 200 HU) festgestellt, die auf eine Ansammlung des peroral verabreichten Kontrastmittels paratracheal links kranial des Schilddrüsenlappens zurückzuführen war. Nach erneuter oraler Kontrastmittelgabe stellten sich kleine Gaseinschlüsse in diesem gleichen Be-

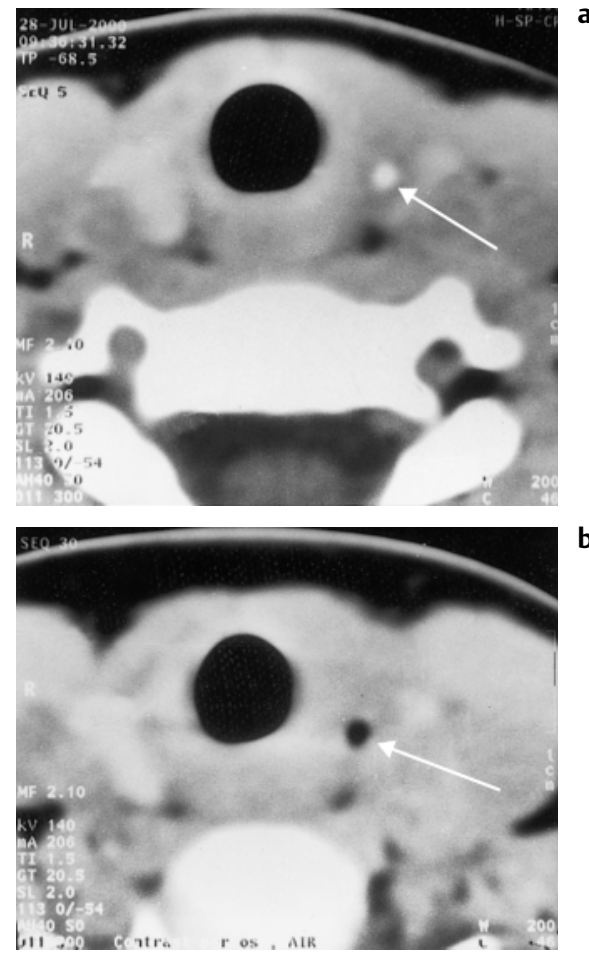

Abb. 2 Computertomographischer Nachweis von Luft li. paratracheal. 
reich dar (Abb. 2 a und $\mathbf{2}$ b).

\section{Fall 2}

Bei einem 15-jährigen Jungen wurde eine akute schmerzhafte Schwellung im linken Halsbereich, begleitet von Schluckbeschwerden, beobachtet. Bei der klinischen Untersuchung wurde eine oberflächliche Raumforderung mit weicher Konsistenz und fluktuierendem Inhalt festgestellt (Abb.3). Die Sonographie zeigte eine ca. $3 \times 2 \mathrm{~cm}$ große abgekapselte hyporeflexive Läsion. Der Patient wurde einer operativen Behandlung zugeführt.

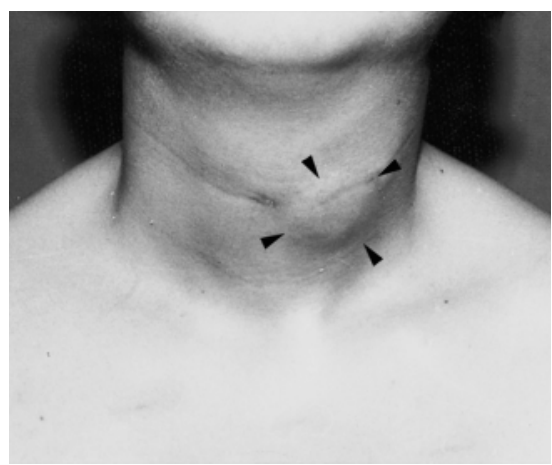

Abb. 3 Akut schmerzhafte Schwellung im li. Halsbereich bei 15-jährigem Jungen.

Intraoperativ wurde die FSP mit Methylenblaukontrastierung nachgewiesen.

\section{Fall 3}

Ein 20-jähriges Mädchen hatte bis vor einem Jahr an einer akuten Thyreoiditis gelitten und war konservativ behandelt worden. Eine Woche vor der stationären Aufnahme wurde eine akute Staphylococcus-aureus-Infektion des Pharynx nachgewiesen. Die klinische Untersuchung zeigte einen vergrößerten und schmerzhaften linken ventralen Schilddrüsenlappen sowie ein ausgeprägtes perithyreoidales Ödem beiTemperaturen um $38^{\circ}$. Die Ultraschalluntersuchung ergab eine asymmetrische Schilddrüse mit vergrößertem linken Lappen, in dem eine inhomogene hyporeflexive Läsion zur Darstellung kam. Eine weitere Läsion wurde im Isthmus festgestellt. In der nativen Computertomographie war die linke Schilddrüse vergrößert, hypodens und inhomogen (Abb. 4). Nach der peroralen Kontrastmittelaufnahme wurde kein Austritt des Kontrastmittels aus dem Pharynx oder der Speiseröhre festgestellt.

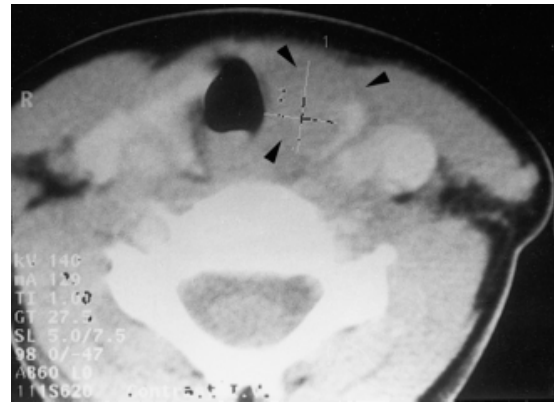

Abb. 4 Computertomographisch dargestellte linksseitige Schilddrüse vergrößert, hypodens und inhomogen.

Eine Verlaufskontrolle nach Antibiotikabehandlung zeigte nach einem Monat einen unauffälligen Uitraschallbefund.

\section{Fall 4}

8-jähriger Junge mit dauerhafter Remission einer akuten Leukämie (seit 18 Monaten). Unter zytostatischer Therapie Entwicklung einer fibrinösen Ösophagitis mit Obstruktion der Speiseröhre. Frustraner Versuch der Insertion einer Magensonde mit Nachweis der Katheterspitze in Höhe BWK7 rechts paravertebral.

Die Computertomographie bestätigte die inadäquate Lage des Katheters im Mediastinum mit Darstellung der Fehllage durch die Entwicklung eines Halsabszesses. Nach Entfernung der Magensonde Nachweis eines perithyreoidalen Abszesses rechts in der Ultraschalluntersuchung. Beginn einer Antibiotikabehandlung mit Überwachung des Kindes über einen Zeitraum von 3 Tagen. Nachdem sich keine Symptome einer Mediastinitis entwickelten, wurde eine direkte Laryngopharyngoskopie durchgeführt. In dieser Untersuchung sowie in einer Kontrastmittelpassage konnte eine vom rechten Sinus piriformis ausgehende Fistel dargestellt werden (Abb. 5). Vier Monate später ist das Kind an den Folgen der Grunderkrankung gestorben. Bei der nachfolgenden Obduktion konnte der persistierende Ductus piriformis nachgewiesen werden.

\section{Diskussion}

Eine mögliche Ursache für die Entstehung eines Halsabszesses oder rezidivierender akuter eitriger Thyreoiditiden ist die kongenitale Fistel des Sinus piriformis (FSP), die erstmalig von Tucker und

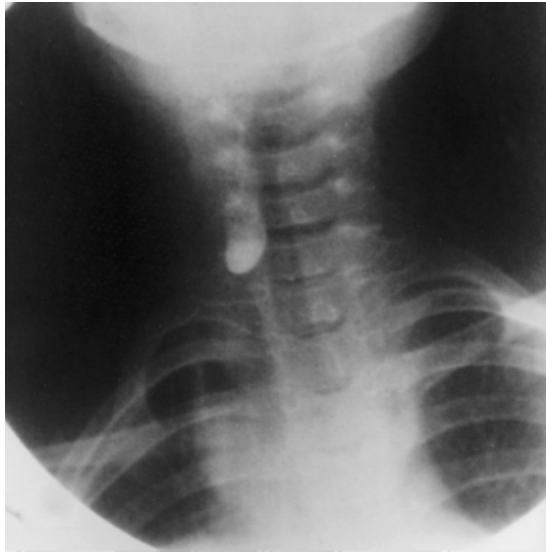

Abb. 5 Perorale Kontrastmittelverabreichung zur Fisteldarstellung vom re. Sinus piriformis ausgehend.

Skolnick im Jahre 1973 (Tucker et al, Trans Am Acad Ophthalmol Otoi 1973; 77:368 - 371) beschrieben worden ist. Der Sinustrakt des IV. Branchialbogens verlässt die Spitze der Pyramidalgrübchen und verläuft unter dem N. laryngeus superior und über dem N. laryngeus recurrens. Der Nachweis von Resten des IV. Branchialbogens ist nur intraoperativ durch Dissektion des Traktes und durch Feststellung seiner anatomischen Verbindung mit dem oberen $\mathrm{N}$. laryngeus möglich (Johnson et al, J Laryngol Otol 1996; 110:391 - 393). Unsere Patienten wiesen die zwei möglichen klinischen Erscheinungsbilder, die mit der FSP verbunden sind, auf: die rezidivierende akute Thyreoiditis oder einen perithyreoidalen Abszess.

Die Resistenz der Schilddrüse gegenüber Infektionen ist im normalen Zustand durch die gute Lymphdrainage, die reiche Blutversorgung und durch den hohen Jodgehalt in der Schilddrüse gesichert. Die akute abszessbildende oder eitrige Thyreoiditis infolge der FSP ist daher eine seltene Erkrankung.

Die Ursache der akuten eitrigen rezidivierenden Thyreoiditis konnte lange Zeit nicht aufgeklärt werden. Häufig konnten aus infizierten Schilddrüsen Bestandteile der normalen oropharyngealen Flora, die auch Anaerobier enthält, isoliert werden. Dieser Befund weist auf eine Kommunikation mit dem Oropharynx hin. Daher wird angenommen, dass eine mögliche Ursache für die rezidivierenden bakteriellen Infektionen der Schilddrüse und des Halses eine Verbindung mit dem piriformen Grübchen ist (Bar- 
Ziv et al, AJF, 1996; 167: 1569 - 1572). Dieser Trakt beginnt an den piriformen Grübchen und endet blind im Schilddrüsenparenchym oder im umliegenden Gewebe. Die häuftigste Infektlokalisation ist das Gewebe um den ventralen linken Oberpol der Schilddrüse oder seltener im Verlauf des Verbindungsorganes. Diese Asymmetrie wird durch die embryonale Entwicklung des vierten Branchialbogens erklärt, aus dem sich die Aorta und A. anonyma entwickeln. Gelegentlich entstehen branchiogene Zysten in den zervikalen Weichteilen oder im Mediastinum. Durch die anatomische Beziehung können die im primär parathyreoidalen Gewebe entstandenen Abszesse eine sekundäre akute Thyreoiditis bzw. eine Infektion des retropharyngealen Gewebes verursachen. Die akute Thyreoiditis wird oft im Rahmen einer akuten Infektion der oberen Atemwege beobachtet, die gewöhnlich im Frühling und im Herbst auftritt. Diese Infektion der Schilddrüse entwickelt sich bei euthyreoten Patienten und unterscheidet sich von den anderen Thyreoiditisformen. Sie tritt häufig vor dem 14. Lebensjahr auf. Eine akute Thyreoiditis oder Halsabszesse wurden aber auch bei jüngeren Kindern beschrieben. Häufig weisen die Patienten eine schmerzhafte Schwellung der unteren Halsweichteile, begleitet von febrilen Temperaturen, auf. Die Erkrankung ist normalerweise einseitig. In den meisten Fällen ist der linke Teil des Halses oder der linke obere Pol der Schilddrüse befallen. Diese Beschwerden werden gewöhnlich als pharyngeale Infektion wahrgenommen. Bei den Neugeborenen und bei den kleinen Kindern kann diese Erkrankung ein „respiratory distress syndrome“ vortäuschen. In Einzelfällen sind Lähmungen der Stimmbänder beschrieben worden.

Rezidive sind häufig und können sogar kurz nach der akuten Entzündung, die mittels Antibiotika oder Drainage behandelt worden ist, auftreten (Fall 3).

Klinisch sind die Fisteln nur schwer nachzuweisen. Die Existenz des Traktes wird mittels endoskopischer Techniken und durch Anwendung der bildgebenden Methoden dargestellt.

Die Ultraschalluntersuchung ist die erste Untersuchung bei klinischem Verdacht auf einen umschriebenen entzündlichen Prozess im Halsbereich. Bei unseren Patienten zeigte sich eine areflexive oder hyperreflexive heterogene Läsion. In den
Fällen der akuten Thyreoiditis (Fall 1 und 3) sind die parathyreoidalen Weichteile vom benachbarten Drüsengewebe nur schlecht abgrenzbar als Ausdruck des Weichteilödems. Bei den Patienten mit einem perithyreoidalen Abszess (Fall 2 und 4) ist die pathologische Formation abgekapselt und die Schilddrüse weist ein normales Parenchym auf. In allen vier Fällen ist es sonographisch nicht möglich gewesen, den Fisteltrakt zu erkennen.

Die Bariumoesophagographie erlaubt eine direkte Darstellung des vom Pharynx ausgehenden Sinustrakts. Wird die Schluckuntersuchung in der akuten Entzündungsphase durchgeführt, verhindert das begleitende Ödem an der Fistelöffnung das Eindringen des Kontrastmittels (Fall 3) (Hristozov et al, Roentgenol Radiol 1999; 38:30-32). Der optimale Zeitpunkt zur Durchführung der Untersuchung ist daher die Rehabilitationsphase nach der Antibiotikabehandlung, d.h. meistens einige Wochen später. Die Schilddrüsen-Szintigraphie gibt keine zusätzliche Information über die Genese der Erkrankung.

Die Computertomographie kann den Entzündungsprozess sicher intra- oder perithyreoidal lokalisieren. Das Ausmaß der Mitbeteiligung der Schilddrüse wird durch undeutliche Grenzen und eine
Dichteabnahme des Schilddrüsenparenchyms deutlich. Abszesse in der Schilddrüse oder im perithyreoidalen Gewebe können an Lufteinschlüssen erkannt werden. Die KM-Verstärkung der Bilder ermöglicht die Abbildung der Abszesskapsel. Darüber hinaus können Gaseinschlüsse im Verlauf des Gangsystems nachgewiesen werden, wenn eine Verbindung zum Pharynx besteht.

Ist eine Hautfistel vorhanden, kann Kontrastmittel direkt injiziert werden. Die Darstellung des Traktes erfolgt dann konventionell röntgenologisch oder computertomographisch. Die direkte Laryngopharyngoskopie ist die optimale endoskopische Technik, da sie die Öffnung des Sinustraktes leichter zeigt als das flexible Endoskop.

Fazit: Wenn Entzündungen oder Abszesse insbesondere auf der linken Seite in den unteren Halsweichteilen rezidivieren, ist differenzialdiagnostisch eine angeborene FSP zu vermuten. Der Nachweis wird durch Ultraschalldiagnostik des Halses und Computertomographie nach der peroralen Verabreichung von Kontrastmittel geführt. Das Hypopharyngooesophagogramm kann in Einzelfällen zusätzliche Informationen über den Befall tieferer Halsstrukturen und des Mediastinums liefern. 